\title{
Evaluación del impacto científico de las tesis doctorales en Ciencias Pedagógicas mediante indicadores cienciométricos
}

\author{
Emilio Ortiz Torres*, María Virginia González Guitián*, \\ Inés Infante Pérez*, Yoan Viamontes Garrido*
}

Resumen: Dentro de las ciencias sociales, las Ciencias Pedagógicas son las que aportan mayor cantidad de tesis doctorales anualmente en Cuba, las cuales abordan problemas científicos de alta pertinencia para la sociedad. Sin embargo, hasta ahora no han sido evaluadas de manera sistemática sus contribuciones científicas una vez presentadas y aprobadas oficialmente mediante herramientas previamente establecidas, por lo que es pertinente la determinación de indicadores cienciométricos que puedan contribuir a la determinación de su impacto científico. En investigaciones anteriores realizadas por los autores de este trabajo, como parte de un proyecto investigativo, se ha constatado que dicha evaluación es insuficiente y desigual, pero sin llegar a determinar indicadores específicos. El objetivo de este artículo es evaluar el impacto científico de las tesis doctorales en Ciencias Pedagógicas.

Palabras clave: impacto científico, evaluación de impacto, indicadores cienciométricos.

\section{The evaluation of scientific impact of doctoral theses in Pedagogical Sciences through scientometric indicators}

Abstract: The doctoral theses in Pedagogical Sciences have a great contribution within social sciences in Cuba, their contents deal with very pertinent scientific problems for the Cuban society. However their scientific contributions have not been systematically evaluated by different specific techniques up to now, for this reason the determination of scientometric indicators is pertinent for the evaluation of their scientific impact. The objective of this article is the evaluation of scientific impact of doctoral theses in Pedagogical Sciences.

Keywords: scientific impact, impact evaluation, scientometric indicators.

* Centro de Estudios sobre Ciencias de la Educación Superior (CECES). Universidad de Holguín Oscar Lucero Moya, Cuba. Correo-e: eortiz@ict.uho.edu.cu, marivi@ict.uho.edu.cu, ines@ict.uho.edu. cu, joan@ict.uho.edu.cu.

Recibido: 30-5-2009; 2. ${ }^{\mathrm{a}}$ versión: 21-10-2009; 3. ${ }^{\mathrm{a}}$ versión: 11-1-2010; aceptado: 28-2-2010. 


\section{Introducción}

La evaluación del impacto de la ciencia y la tecnología constituye una necesidad estratégica para constatar el desarrollo de un país, de su política científica y de su gestión en función de la sociedad y de los seres humanos que conviven en ella. Sobre su pertinencia e importancia se pronuncian varios autores que investigan y publican sobre este aspecto (Bocco, 2002; Moñux y otros, 2005; Villaveces y otros, 2005).

Por la propia misión de las universidades es imprescindible que su producción científica sea objeto de reflexión y evaluación debido a los imperativos del desarrollo social, y sobre ello diferentes autores se han referido (Agudelo y otros, 2003; Cortes, 2007; García, 2006).

Dentro de las investigaciones científicas las tesis doctorales tienen un lugar muy destacado en las universidades porque, además de constituir un requisito para obtener un grado científico, aportan resultados relevantes. En Cuba el doctorado y la investigación están altamente integrados como programas y como proyectos, ya que las tesis doctorales constituyen salidas importantes que favorecen el mayor y más rápido impacto de los resultados de investigación. La tendencia es que los proyectos de investigación desborden ampliamente el doctorado y sean necesarios varios doctorados que aporten los resultados comprometidos (Llanio y otros, 2007).

El patrón de calidad de las tesis doctorales, asumido por la Comisión Nacional de Grados Científicos de la República de Cuba, se refiere al impacto social de sus resultados, dentro del cual están los aportes científicos a la especialidad en cuestión, las publicaciones de sus resultados parciales o finales, el liderazgo científico de sus egresados, así como la obtención de premios y reconocimientos. Sin embargo, no ha constituido hasta el presente una práctica la evaluación recurrente de su impacto como medio para constatar dicha calidad, aunque como parte de las exigencias para la culminación e las tesis doctorales en Cuba se establecen legalmente determinados requisitos de publicación, ya sea en revistas científicas como en eventos (Moñux y otros, 2005).

Dentro de las especialidades que integran las ciencias sociales, es en las ciencias pedagógicas donde más doctores se forman anualmente, pero hasta ahora no han sido evaluadas sus contribuciones científicas una vez presentadas y aprobadas oficialmente mediante herramientas científicas previamente establecidas, por lo que es pertinente la determinación de indicadores cienciométricos que puedan contribuir a la evaluación de su impacto científico.

En constataciones realizadas previamente se ha determinado que dicha evaluación es insuficiente y desigual (Ortiz y otros, 2007, 2008). Por tanto, el propósito de este artículo es evaluar el impacto científico de las tesis doctorales en ciencias pedagógicas, sobre la base de los siguientes indicadores cienciométricos previamente seleccionados: 
1. Índice de citación: frecuencia con que es citada la tesis en otras tesis o en otras investigaciones, monografías, libros o en artículos científicos publicados posteriormente a su defensa.

2. Índice de visibilidad: presencia de los resultados parciales o finales de la tesis doctorales publicados en diferentes revistas científicas y sitios académicos de Internet y su fácil acceso.

3. Impacto económico-social: premios, reconocimientos, distinciones, galardones o recompensas otorgadas a los resultados parciales o finales de las tesis por parte de diferentes entidades, organismos u organizaciones como constancia de su aporte científico y/o social.

4. Publicación en revistas arbitradas: artículos científicos derivados de las tesis, ya sea de sus resultados parciales o totales que han sido publicados en revistas que poseen un consejo editorial, el cual controla la calidad de artículos.

5. Publicación en bases de datos de prestigio internacional: sitio específico en que están publicados los resultados parciales o finales de la tesis, como por ejemplo en EBSCO, LATINDEX, la Biblioteca Virtual de las Organización de Estados Iberoamericanos (OEI), entre otros.

6. Publicación de libros y monografías como continuidad de la tesis doctoral: ya sea en base magnética o en papel, pero con la exigencia de que posean ISBN.

7. Participación en eventos nacionales e internacionales: presentación de resultados parciales o finales de la tesis en Cuba o en el extranjero y certificación oficial de su presentación.

8. Resultados introducidos en la práctica social: la constancia de que el aporte práctico de la tesis ha sido aplicado mediante la existencia de avales por parte de introductores que lo certifiquen.

\section{Metodología empleada}

En la provincia de Holguín se ha venido produciendo un incremento sostenido de las investigaciones pedagógicas desde la década de los años ochenta hasta la fecha, debido a la realización de proyectos de investigación muy pertinentes, cuyos salidas principales han sido la realización de tesis doctorales, con el aporte de conocimientos científicos valiosos, así como el incremento de doctores en dichas ciencias.

Para la aplicación de estos indicadores se utilizó la información obtenida de varios centros de educación superior de esta provincia, así como de la delegación territorial del Ministerio de Ciencia, Tecnología y Medio Ambiente.

Se utilizaron varios métodos y técnicas investigadoras, tales como la revisión del contenido de las tesis, los premios otorgados al nivel provincial, la búsqueda en Internet de la divulgación de los resultados científicos derivados de ellas, así como entrevistas individuales a sus autores, tutores, jefes de proyectos y directivos. 


\section{Resultados}

Se lograron censar todas las tesis doctorales en ciencias pedagógicas realizadas en el territorio holguinero desde 1988 hasta el 2008, que fueron 107. De la población total se muestrearon aleatoriamente 74 (69\%) para valorar en su contenido la divulgación de los resultados parciales durante su realización en revistas y en eventos científicos nacionales e internacionales y los premios recibidos al nivel provincial.

La tabla I refleja la frecuencia en que dichas tesis fueron realizadas en el período de 1988 hasta el 2008, lo que evidencia un crecimiento sostenido sobre todo a partir del año 2001, para un total de 107 tesis presentadas y aprobadas oficialmente.

\section{TABLA I}

Comparación entre las tesis doctorales en ciencias pedagógicas realizadas y premiadas en el período de 1988-2008

\begin{tabular}{c|c|c}
\hline Periodos & Tesis defendidas & Tesis premiadas \\
\hline $1988-1995$ & 3 & 3 \\
\hline $1996-2000$ & 5 & 2 \\
\hline $2001-2005$ & 43 & 6 \\
\hline $2006-2008$ & 56 & 2 \\
\hline
\end{tabular}

Las causas se encuentran en las políticas científicas finiseculares para el desarrollo significativo de las investigaciones científicas en los centros de educación superior, con la determinación de prioridades de investigación y su consecuente aprobación oficial y financiamiento a través de proyectos de investigación territoriales, ramales y nacionales que incluían tesis doctorales asociadas, los cuales comienzan a aportar resultados concretos a principios del siglo xxI.

La Academia de Ciencias de Cuba (ACC), desde el año 1990, instituyó oficialmente el premio que se otorga al nivel nacional y/o provincial a aquellas investigaciones que aportan resultados científicos novedosos y que hayan logrado introducirlos en la práctica social mediante los avales oficiales emitidos por las entidades introductoras, el cual es denominado Premio de la Academia de Ciencias de Cuba (Premio ACC).

La tabla I compara la frecuencia de realización de las tesis doctorales en ciencias pedagógicas con aquéllas a las que les fue otorgado el Premio ACC al nivel provincial desde 1988 hasta 2008. Los datos contrastan debido a la pobre premiación lograda a pesar del incremento sostenido en el número de tesis a partir del período 2001-2005.

Para determinar las causas de esta desproporción se aplicaron entrevistas individuales a los autores y tutores, jefes de proyectos y directivos en las que se 
les pedía que valoraran por qué no habían sido premiadas. Las respuestas evidenciaron los siguientes criterios:

- Para los tutores y autores no constituyó una preocupación la gestión de un premio, ni durante la realización de las tesis, ni posteriormente.

- Los jefes de proyectos y directivos tampoco planificaron ni gestionaron los posibles premios asociados a las tesis.

De lo que se infiere la inexistencia de una planificación estratégica para la gestión y obtención de dichos premios, así como tampoco la introducción oportuna de los resultados científicos en la práctica social.

La tabla II resume el número de artículos científicos publicados con los resultados parciales de las tesis en revistas arbitradas, reconocidas oficialmente, de gran visibilidad y que forman parte de bases de datos de prestigio internacional. Los datos muestran que el 78\% no publicó en ninguna de ellas, el 19\% uno o dos y el 3\%, tres o cuatro.

\section{TABLA II}

Publicación de los resultados parciales en revistas arbitradas $y$ de visibilidad

\begin{tabular}{l|c|c}
\hline & Publicaciones revistas arbitradas & $\mathbf{\%}$ \\
\hline Ninguna & 58 & 78 \\
\hline $1-2$ & 14 & 19 \\
\hline $3-4$ & 2 & 3 \\
\hline
\end{tabular}

Para indagar en las causas de estos pobres resultados se aplicaron entrevistas individuales a los autores y tutores de las tesis y se constató que en la mayoría de los casos sus intereses estuvieron en la presentación de ponencias en eventos científicos en la medida que iban obteniendo dichos resultados científicos parciales y no en la publicación de artículos científicos en este tipo de revistas, por el nivel de exigencia que plantean los árbitros para su aceptación. Los que sí lograron publicar fueron debido a decisiones individuales por la importancia personal que le concedieron al proceso de realización de sus tesis doctorales.

La tabla III expone la divulgación de resultados parciales como ponencias en eventos científicos nacionales con determinado nivel de selectividad para lograr la participación por la calidad de ellas. El 61\% de las tesis no dio lugar a ninguna, el $21 \%$ una o dos y el $18 \%$ tres o cuatro ponencias.

Las entrevistas individuales a tutores y autores reflejaron grandes diferencias de criterios en cuanto a la prioridad atribuida a la presentación de sus resultados parciales en este tipo de eventos, pues la mayoría no consideró pertinente la confrontación pública ante la comunidad científica de dichos resultados; solo una 
TABLA III

Presentación de ponencias en eventos científicos nacionales

\begin{tabular}{l|c|c}
\hline & Ponencias eventos científicos nacionales & $\mathbf{\%}$ \\
\hline Ninguna & 45 & 61 \\
\hline $1-2$ & 15 & 21 \\
\hline $3-4$ & 13 & 18 \\
\hline
\end{tabular}

minoría lo valoró como necesario para el propio proceso de realización de las tesis.

La tabla IV muestra la participación en eventos internacionales, cuya asistencia es aun más selectiva y exigente. La gran mayoría (57\%) no asistió nunca, el $20 \%$ en uno o dos, el $16 \%$ en tres o cuatro y el $7 \%$ en cinco o más.

TABLA IV

Presentación de ponencias en eventos científicos internacionales

\begin{tabular}{l|c|c}
\hline & Ponencias eventos científicos internacionales & $\mathbf{\%}$ \\
\hline Ninguna & 42 & 57 \\
\hline $1-2$ & 15 & 20 \\
\hline $3-4$ & 12 & 16 \\
\hline 5 o más & 5 & 7 \\
\hline
\end{tabular}

Las entrevistas individuales a autores y tutores reiteran la misma consideración de los gráficos anteriores sobre la poca importancia que le confieren ambos para divulgar sus aportes en dichos eventos.

Estos datos confirman la insuficiente gestión del impacto científico durante la realización de las tesis doctorales en ciencias pedagógicas y posteriormente, debido a su no consideración como prioritarios por parte de tutores y autores, a pesar de la pertinencia y novedad de los conocimientos aportados.

\section{Conclusiones}

El análisis de los resultados obtenidos evidencia las dificultades existentes en la gestión de la calidad durante el proceso de su realización de las tesis y su divulgación por responsabilidad de directivos, tutores y autores.

Los datos de las entrevistas individuales aplicadas a ellos revelaron que, aunque la pertinencia de los temas de tesis es muy alta, no ha constituido para ellos una prioridad la certificación de la cientificidad de los resultados por parte de la 
comunidad científica nacional e internacional, ni tampoco se ha erigido en una exigencia oficial de inexcusable cumplimiento.

Por lo que se ha adquirido conciencia del problema por parte de todos los implicados y, junto con la Comisión Nacional de Grados Científicos de la República de Cuba, se han tomando en el territorio holguinero y en el país varias medidas para garantizar un producto final en correspondencia con el patrón de calidad de las tesis doctorales en Cuba, lo que ha provocado que la situación comience a revertirse a partir del año 2009.

La evaluación del impacto científico de las tesis doctorales en ciencias pedagógicas siempre constituirá una necesidad perentoria debido a su gran pertinencia y a las demandas actuales para que contribuyan de manera real y efectiva al perfeccionamiento en la formación de las nuevas generaciones en las condiciones sociales actuales.

Al comparar estos resultados con trabajos previos realizados por otros autores se constata que, si bien en el campo de las ciencias naturales y exactas no constituye una novedad la aplicación de indicadores cienciométricos en la evaluación del impacto científico, en las ciencias sociales no constituye hasta ahora una tradición hacerlo, por lo que este trabajo intenta aportar en este sentido y continuar en esta senda promisoria.

Los indicadores propuestos, si bien son conocidos y utilizados en el plano internacional, no han sido nunca utilizados de manera sistemática para evaluar tesis en esta especialidad. Su eficacia radica en la información fidedigna que ofrece para las evaluaciones correspondientes de impacto científico a todas las tesis doctorales en estas ciencias.

Estos indicadores son factibles de aplicar de manera inmediata sin erogar grandes recursos materiales o financieros, solamente a partir de la revisión individual de cada tesis, de la entrevista individual a tutores y aspirantes, de la búsqueda en Internet, en revistas y bases de datos. La utilización de estos indicadores de manera oportuna y sistemática debe formar parte del proceso permanente de gestión de la calidad de las ciencias pedagógicas.

\section{Referencias bibliográficas}

Agudelo, D.; Breton López, J.; Ortiz Recio, G.; Poveda Vera, J.; Teva, I.; Valor Segura, I., y Vico, C. (2003): Análisis de la productividad científica de la Psicología Española a través de las tesis doctorales. Psicothema, 15 (4), 595-609.

Bocco, G. (2002): Evaluación del impacto científico. GEOS, Unión Geofísica Mexicana, A. C. La Jornada, 27 de marzo (supl.).

Cortes, D. (2007): Medir la producción científica de los investigadores universitarios: la bibliometría y sus límites. Revista de la Educación Superior, vol. XXXIV (142): 43-65, Madrid.

García, J. L. (2006): Investigaciones y doctorados en la universidad cubana. Reflexiones en el nuevo siglo. En Colectivo de Autores. Gestión de ciencia y tecnología en las universidades. La experiencia cubana. La Habana: Editorial Félix Varela. 
Llanio Martínez, G.; Peniche Cobas, C., y Rodríguez Pendás, M. (2007): Los caminos hacia el doctorado en Cuba, p. 30. La Habana: Ministerio de Educación Superior.

Moñux Chércoles, D.; Miranda Escolar, B.; Mendizábal Aleixandre, G., y Gómez González, F. J. (2005): Condicionantes políticos y problemas metodológicos en la evaluación de impacto social de las políticas de I+D e innovación. Revista CTS, v. 2 (3), 173-200.

Ortiz Torres, E.; Infante Pérez, I.; González Guitián, M. V., y Viamontes Garrido, Y. (2007): Impacto de las tesis doctorales en ciencias pedagógicas en el territorio holguinero. Revista Electrónica Pedagogía Universitaria, v. XII (5). La Habana: Ministerio de Educación Superior.

Ortiz Torres, E.; Infante Pérez, I.; González Guitián, M. V., y Viamontes Garrido, Y. (2008): Impacto de las tesis doctorales en ciencias sociales en el territorio holguinero. Memorias de la Convención Internacional Universidad 2008, La Habana.

Batista Rodríguez, A. (2005): Impacto social de la ciencia y la tecnología en Cuba: una experiencia de medición a nivel macro. Revista CTS, v. 2 (4): 147-171, Madrid.

Villaveces, J. L.; Antonio Orozco, L.; Lucía Olaya, D.; Chavarro D., y Suárez, E. (2005): ¿Cómo medir el impacto de las políticas de ciencia y tecnología? Revista CTS, v. 2 (4): 125-146, Madrid. 